\title{
Prevalence of leptospirosis and toxoplasmosis: A study of rodents and shrews in cultivated and fallow land, Morogoro rural district, Tanzania
}

GEORGIES F. MGODE ${ }^{1 *}$, ABDUL S. KATAKWEBA ${ }^{1}$, GINETHON G. MHAMPHI', FRANK FWALO², MOHAMED BAHARI ${ }^{2}$, MASHAKA MDANGI, ${ }^{1,3}$, BUKHETI S. KILONZO ${ }^{1}$ and LOTH S. MULUNGU ${ }^{1}$

${ }^{1}$ Pest Management Centre, Sokoine University of Agriculture, P.O. Box 3110, Morogoro, Tanzania

${ }^{2}$ Department of Veterinary Microbiology and Parasitology, Sokoine University of Agriculture, Morogoro, Tanzania

${ }^{3}$ Ministry of Agriculture Training Institute, Ilonga, P.O. Box 66, Kilosa, Tanzania

Abstract: Leptospirosis and toxoplasmosis are among understudied zoonotic diseases that are also not diagnosed routinely in Tanzania. Humans get leptospirosis and toxoplasmosis through contact with an environment contaminated with Leptospira bacteria and Toxoplasma protozoa from reservoir hosts, which are rodents and cats, respectively. The objective of this study was to determine the prevalence of Leptospira and Toxoplasma infections in rodents and shrews in Mikese area of Morogoro Rural District in eastern Tanzania. A total of 89 rodents and one shrew from cultivated and fallow land were tested for leptospirosis using six Leptospira serovars: Sokoine, Kenya, Canicola, Lora, Hebdomadis and Pomona. Toxoplasmosis was determined in 46 rodents brain smears. The prevalence of leptospirosis was $25.8 \%$, and Leptospira serovar Sokoine was the most prevalent serovar (16.9\%). Toxoplasma was detected in one rodent (2.17\%) individual while three rodent individuals had Toxoplasma-like parasites hence were considered suspect positive. Findings suggest potential existence of human leptospirosis which needs to be further investigated. Public awareness of leptospirosis and toxoplasmosis should be promoted and their diagnosis considered in patients in health care facilities.

\section{Keywords: Leptospirosis, toxoplasmosis, land use, zoonosis, rodents, shrews, Tanzania}

\section{Introduction}

Rodents are reservoirs of various emerging infectious diseases including leptospirosis caused by Leptospira bacterium and toxoplasmosis caused by Toxoplasma gondii (Jones et al., 2001). These diseases are among the understudied rodent borne zoonotic diseases in Africa. Previous studies in rodents in Tanzania including Morogoro urban areas show a high prevalence of leptospirosis. Prevalence of leptospirosis in cattle in pastoral areas of Tanzania has been reported as $5.6 \%$ (Machang'u et al., 1997) while among cattle sampled at livestock market as $51 \%$ (Swai \& Schoonman, 2012). A prevalence of leptospirosis of $37 \%$ has been reported in dogs in Moshi (Machang'u et al., 1997). A study among patients with fever admitted at a hospital in northern Tanzania reported a leptospirosis prevalence of 9\% (Biggs et al., 2011).

A high prevalence of toxoplasmosis (52.2\%) has been reported among livestock keepers in Tanga, north-eastern Tanzania (Swai \& Schoonman, 2009). A recent study in Mwanza in northern Tanzania has shown that $30.9 \%$ of pregnant women were infected with Toxoplasma (Mwambe et al., 2013), whereas in Dar es Salaam a prevalence of 35\% has been reported (Doehring et al., 1995). Relatively higher prevalence of toxoplasmosis has been reported elsewhere in the world. The prevalence of toxoplasmosis in child-bearing women in rural Sudan is even higher ranging between $60.7 \%$ and $87.3 \%$ (Mohamed et al., 2009). In the USA it is reported that $85 \%$ of childbearing women have toxoplasmosis (Jones et al., 2001). This shows that toxoplasmosis is an important zoonosis. Humans get leptospirosis and toxoplasmosis through contact with environment contaminated with Leptospira and Toxoplasma pathogens mainly from rodents and cats, respectively (Babudieri, 1958; Frenkel \& Ruiz, 1981; Hill \& Dubey, 2002).

Human and other animals such as rodents excluding cats are intermediate hosts of Toxoplasma gondii. Intermediate host animals infected with T. gondii changes their normal

\footnotetext{
*Correspondence: Georgies F. Mgode; E-mail: gfmgode@hotmail.com; gmgode@suanet.ac.tz
} 
behaviour towards predators. For example, rodents infected with $T$. gondii tend not to fear cats (House et al., 2011; Ingram et al., 2013; Flegr et al., 2002; Flegr et al., 2011; Berdoy et al., 2000), which are natural enemies of rodents. Toxoplasmosis infections in rodents may increase rodentpredators associations and increase public health threats especially in areas where rodents are eaten across Tanzania, Africa, Asia and Latin America (Ter Meulen et al., 1996; Khiem \& Chien, 2003; Assogbadjo et al., 2005; Barragán et al. 2007).

There is limited information on the prevalence of both leptospirosis and toxoplasmosis in rodents and other intermediate host animals in Tanzania. This study therefore was carried out to: (i) determine the prevalence of leptospirosis and toxoplasmosis in rodents and shrews in Morogoro rural, Tanzania; and (ii) to compare the prevalence of the disease in animals from cultivated and fallow land to establish any association between disease prevalence with habitat type.

\section{Materials and Methods}

\section{Study site}

This study was carried out in Mikese village ( $37^{\circ} 53^{\prime} 60^{\prime} \mathrm{E}$; $6^{\circ} 46^{\prime} \mathrm{S}$ ) located in Morogoro Rural District in Tanzania some 30 kilometres east of the Morogoro Municipality, along the Morogoro Dar es Salaam highway. The annual rainfall ranges from 700 to $1000 \mathrm{~mm}$. The short rainy season starts in mid-October and ends in December while the long rains start in February and ends in mid May. The dry season extends from June to October. The annual average maximum and minimum temperatures are $26^{\circ} \mathrm{C}$ and $21^{\circ} \mathrm{C}$, respectively. Soils are acidic lithosols and ferralitic latosols with deeper deposits of ferruginous sandy clay. Mikese villagers depend mostly on rain-fed agriculture and maize is the main crop. Other crops cultivated include beans, soybeans and horticultural crops.

\section{Animal trapping}

Live rodents and shrews were captured in cultivated and fallow land fields measuring $70 \times 70 \mathrm{~m}$ each from August 2012 through May 2013. Each field consisted of seven parallel lines, 10m apart, with seven trapping stations per line, also 10m apart, making a total of 49 trapping stations per grid. One Sherman LFA live trap $(8 \times 9 \times 23 \mathrm{~cm}$, H.B. Sherman Traps Inc., Tallahassee, FL, U.S.A.) was placed at each trapping station and all were set for three consecutive nights at intervals of four weeks. Traps were baited with peanut butter mixed with maize bran/maize flour, set in the afternoon, and inspected in the morning. All captured animals were taken for subsequent analyses (removal trapping). The distance between these two fields was approximately $2 \mathrm{~km}$.

\section{Processing of captured animals}

All the captured animals were taken to the field laboratory, sex determined, and identified to species level as described by Kingdon (1997). Blood samples were collected from orbital vein and through heart puncture after anaesthetizing the animals with di-ethyl ether. Serum samples for serological detection of Leptospira antibodies were separated from blood by centrifugation. Rodent brain was preserved in $80 \%$ ethanol for preparation of brain impression smears for determination of Toxoplasma pathogens.

\section{Detection of Leptospira infection}

Antibodies against Leptospira were determined using microscopic agglutination test (MAT) (Cole et al., 1973; Goris \& Hartskeerl, 2013). Six Leptospira serovars were selected for use as live antigen in MAT. These included reference serovars and local serovars isolated from cattle and rodents in Morogoro areas, namely, serovar Sokoine, Kenya, Lora, Canicola (Machang'u et al., 2004; Mgode et al., 2006; Ahmed et al., 2006), Hebdomadis and Pomona. The selected serovars were grown into fresh Leptospira Ellinghausen and McCullough, modified by Johnson and Harris (EMJH) 
culture medium for 4-7 days while monitoring for growth density and contamination using a darkfield microscope.

\section{Detection of Toxoplasma parasites}

A total of 46 rodents were examined for toxoplasmosis. Brain impression smears were made onto clean microscope slides and left to dry at room temperature. Brain suspension splash smears were prepared by putting the brain into a clean test tube containing phosphate buffered saline ( $\mathrm{pH}$ 7.0) and homogenized using sterile glass rods to obtain brain suspension. A drop of brain suspension was transferred using sterile Pasteur pipette onto clean microscope slides. The smears were left to dry in air and thereafter stained with 1:10 Giemsa solution for 3omin. Smears were washed with running tap water and dried in air before examining for Toxoplasma parasites under light microscope (1000 x magnification).

\section{Data analysis}

The prevalence of the disease in animals from cultivated and fallow land was compared through descriptive analysis (Microsoft Excel, 2013) to determine whether there was association between disease prevalence with habitat type by examining the proportions of positives animals from the two fields out of the total positives. A Leptospira serovar Sokoine that was most prevalent in both sites was used to determine this association.

\section{Results}

\section{Rodents and shrews}

A total of 89 rodents belonging to five rodent genera and one shrew genus were collected from cultivated and fallow land (Table 1). Mastomys natalensis was the most predominant species contributing to $79 \%$ of total captures.

Table 1: Composition of rodent species and shrews in the study area

\begin{tabular}{llll}
\hline Type & Genus/species & Number & Percent composition \\
\hline Rodents & Mastomys natalensis & 71 & 78.88 \\
& Lemniscomys sp. & 6 & 6.66 \\
& Acomys sp. & 6 & 6.66 \\
& Gerbilliscus vicinus & 5 & 5.55 \\
Shrews & Nannomys sp. & 1 & 1.11 \\
Total & Crocidura sp. & 1 & 1.11 \\
\hline
\end{tabular}

\section{Prevalence of Leptospirosis}

Twenty-three rodents out of 89 and one shrew tested positive against Leptospira serovars Sokoine, Lora, Kenya, Canicola and Hebdomadis. The overall prevalence of leptospirosis in rodents determined using six Leptospira serovars was $25.8 \%$. Leptospira serovar Sokoine was more prevalent in these sites (Table 2) with 15 leptospirosis positive animals coming from both cultivated and fallow land. Eight of the 15 positive animals for serovar Sokoine were from cultivated land (53.3\%) and the remaining 7 were from fallow land (46.6\%). Other serovars reacted with fewer animals whereas serovar Pomona was not detected. A single shrew (Crocidura sp.) was positive to serovar Canicola.

Majority of the leptospirosis positive rodents had low antibodies titres (1:20 and 1:40) which are below the current adopted cut-off point of 1:160. A Crocidura sp. (shrew) was only positive to serovar Canicola with low titre (1:20). Such a low titre was also observed from one rodent species against serovar Canicola. A relatively high titre (1:80) was found against serovar Sokoine and Lora in two rodents. The distribution of antibodies levels in rodents against the six Leptospira serovars is shown in Table 3. 
Table 2: Prevalence of six Leptospira serovars in rodents

\begin{tabular}{llll}
\hline $\begin{array}{l}\text { Leptospira } \\
\text { serovars tested }\end{array}$ & $\begin{array}{l}\text { Number of } \\
\text { animals }\end{array}$ & Leptospirosis positive (1:20 - 1:80 titres)* & Serovar prevalence \\
\hline Sokoine & 89 & 15 & $16.9 \%$ \\
Lora & 89 & $8(3)$ & $9 \%$ \\
Kenya & 89 & $2(1)$ & $2.2 \%$ \\
Canicola & 89 & $2(1)$ & $2.2 \%$ \\
Hebdomadis & 89 & 1 & $1.1 \%$ \\
Pomona & 89 & 0 & 0.0 \\
\hline * Number of specimens in brackets reacted with more than one Leptospira serovar including serovar Sokoine (cross \\
reactions) hence are not included in overall prevalence (23 positive animals out of 89).
\end{tabular}

Table 3: Antibody titres of rodents $(n=89)$ and shrew $(n=1)$ tested with MAT including six Leptospira serovars

\begin{tabular}{lllllll}
\hline \multirow{2}{*}{ Titre } & \multicolumn{2}{l}{ Leptospira serovars } & & & \\
\cline { 2 - 7 } & Sokoine & Lora & Kenya & Canicola & Hebdomadis & Pomona \\
\hline $1: 20$ & 13 & 4 & 2 & 2 & 1 & 0 \\
$1: 40$ & 1 & 3 & 0 & 0 & 0 & 0 \\
$1: 80$ & 1 & 1 & 0 & 0 & 0 & 0 \\
\hline Total & 15 & 8 & 2 & 2 & 1 & 0 \\
\hline
\end{tabular}

The predominant rodent species $M$. natalensis contributed more to leptospirosis positive animals: M. natalensis ( $n=18)$, Lemniscomys sp. $(n=3)$, Acomys sp. $(n=2)$, and one Crocidura sp. (shrew). Serovar Sokoine was detected most in M. natalensis $(n=12)$, Lemniscomys sp. $(n=2)$ and Acomys $(n=1)$, whereas serovar Lora was also detected most in M. natalensis $(n=6)$, and in 1 Acomys and Lemniscomys sp.

\section{Prevalence of Toxoplasmosis}

There was one positive rodent out of 46 examined for Toxoplasma parasites in rodent brain smears (2.17\%). Three other rodents were suspects requiring further analysis with other methods to confirm the positivity. The rodent with Toxoplasma was Lemniscomys sp. collected from fallow land. Despite their abundance, $M$. natalensis (Table 1) was negative for toxoplasmosis. A Crocidura sp. (shrew) was also negative for toxoplasmosis.

\section{Discussion}

Findings of this study show a high prevalence of leptospirosis in rodents and shrews from both cultivated and fallow areas. Eight rodents with leptospiral antibodies were from fallow land whereas seven were from cultivated land. This relatively equal distribution of positive rodents and shrews assessed using serovar Sokoine which was most prevalent in both sites suggests that rodents and shrews movements are not restricted in these sites and they could efficiently transmit diseases in both areas as was expected. Leptospira serovar Sokoine was the most prevalent in positive rodents. This serovar has been isolated in cattle and rodents from Morogoro areas (Mgode et al., 2006; Ahmed et al., 2006) hence these findings suggest that serovar Sokoine is potentially the widely circulating Leptospira in animals in this region.

Other serovars which reacted in the MAT were Leptospira serovars Lora also reported in rodents from Morogoro areas; serovar Kenya from African giant rats, serovar Canicola which reacted with a Crocidura is specific for dogs, whereas serovar Hebdomadis has wide range of hosts and serovar Pomona is common in pigs. Seropositivity for the five Leptospira serovars was characterized by low antibody titres with two animals demonstrating a relatively higher titre, which is also below the 1:160 cut-off point adopted from European study (Goris et al., 2012). These 
results suggest urgent need of reviewing the cut-off-point of leptospirosis diagnosis by microscopic agglutination test in Tanzania and other countries in Africa. The need for establishing the actual cut-off titres for this region is especially important due to differences environmental factors and diversity of reservoir hosts.

Lower titres may suggest chronic leptospirosis infections demonstrated by low levels of $\lg G$ that can be below the detection threshold of MAT, or infection with Leptospira serovars related to the reacting serovars included in the MAT. Serovars belonging to same serogroup tend to cross react and levels of cross-reactions depict their relatedness (Dikken \& Kmety, 1978; Faine, 1982). Serovar Sokoine belongs to serogroup Icterohaemorrhagiae widely reported in eastern and central Africa (Faine et al., 1999).

Serovar Sokoine cross reacts with several members of L. kirschneri species serogroup Icterohaemorrhagiae such as serovar Mwogolo, Ndahambukuje and Ndambari first described in the Democratic Republic of Congo (Faine et al., 1999). These findings calls for further studies including PCR detection of leptospiral DNA (Mgode et al., 2005) and isolation of leptospires from rodents in Mikese area to enhance identification of the causative serovars. Such information is necessary for future surveillance and development of list of local Leptospira serovars for human leptospirosis diagnosis.

Low prevalence of serovars Kenya, Canicola, Hebdomadis and absence of serovar Pomona in this study suggest host-specificity. For example, serovar Kenya which is common in Morogoro is widely found in African giant pouched rats (Cricetomys sp.) (Kranendork et al., 1968; Machang'u et al., 2004) was not investigated in this study.

The prevalence of toxoplasmosis in the study area is generally low. However, this could be an underestimate considering the crude method used (Giemsa staining) and relatively small sample size. Further studies involving robust serological and molecular detection of $T$. gondii antibodies and DNA (Desmonts et al., 1980; Homan et al., 2000; Burg et al., 1989) are needed to establish actual prevalence.

It can be concluded that leptospirosis is harboured by different species of rodents and shrew species found in Mikese. The rodents and shrew from this area have high prevalence of leptospirosis and a relatively lower prevalence of toxoplasmosis. There is a need to increase public awareness of rodent-borne diseases such as leptospirosis and toxoplasmosis that are likely to be transmitted to humans engaged in agricultural activities in this area. The burden of leptospirosis in humans and domestic animals needs to be determined considering that people engaged with farming in the study area come from different parts of Tanzania including areas where rodents are eaten as food source that increase risks of rodent-borne infections. Further investigation on prevalence of toxoplasmosis should be carried out using serological or molecular methods that are robust than microscopic examination of $T$. gondii. The diagnosis of leptospirosis, toxoplasmosis and other neglected zoonoses should be given priority in Tanzania to improve public health.

\section{Acknowledgements}

The authors would like to thank Mr. Ramadhani Iddi, Khalid Kibwana for field assistance, and farmers for their cooperation. This study received financial assistance from the Tanzania Commission for Science and Technology through the Cat Urine Odour Project.

\section{References}

Ahmed, N., Devi, S.M., Valverde Mde, L., Vijayachari, P., Machang'u, R.S., Ellis, W.A. \& Hartskeerl, R.A. (2006) Multilocus sequence typing method for identification and genotypic classification of pathogenic Leptospira species. Annals of Clinical Microbiology and Antimicrobials 5, 28. 
Assogbadjo, A.E., Codjia, J.T.C., Sinsin, B., Ekue M.R.M. \& Mensah, G.A. (2005) Importance of rodents as a human food source in Benin. Belgian Journal of Zoology 135, 11-15.

Babudieri, B. (1958) Animal reservoirs of leptospires. Annals of New York Academy of Sciences 70, 393-413.

Barragán, F., Retana, O.G. \& Naranjo, E.J. (2007) The rodent trade of Tzeltal Indians of Oxchuc, Chiapas, Mexico. Human Ecology 35, 769-773.

Berdoy, M., Webster, J.P. \& Macdonald, D.W. (2000) Fatal attraction in rats infected with Toxoplasma gondii. Proceedings of the Royal Society of London. Series B: Biological Sciences 267 (1452), 1591-1594.

Biggs, H.M., Bui, D.M., Galloway, R.L., Stoddard, R.A., Shadomy, S.V., Morrissey, A.B., Bartlett, J.A., Onyango, J.J., Maro, V.P., Kinabo, G.D., Saganda, W. \& Crump, J.A. (2011) Leptospirosis among hospitalized febrile patients in northern Tanzania. American Journal of Tropical Medicine and Hygiene 85, 275.

Burg, J.L., Grover, C.M., Pouletty, P. \& Boothroyd, J.C. (1989) Direct and sensitive detection of a pathogenic protozoan, Toxoplasma gondii, by polymerase chain reaction. Journal of Clinical Microbiology 27, 1787-1792.

Cole, J.R., Sulzer, C.R. \& Pursell, A.R. (1973) Improved microtechnique for the leptospiral agglutination test. Applied Microbiology 25, 976-980.

Desmonts, G. \& Remington, J.S. (1980) Direct agglutination test for diagnosis of Toxoplasma infection: Method for increasing sensitivity and specificity. Journal of Clinical Microbiology $11,562-568$.

Dikken, H. \& Kmety, E. (1978) Serological typing methods of leptospires. Methods in Microbiology 11, 259-307.

Doehring, E., Reiter-Owona, I., Bauer, O., Kaisi, M., Hlobil, H., Quade, G., Hamudu, N.A. \& Seitz, H.M. (1995) Toxoplasma gondii antibodies in pregnant women and their newborns in Dar es Salaam, Tanzania. American Journal of Tropical Medicine and Hygiene 52, 546-548.

Faine, S. (Ed.) (1982) Guidelines for the Control of Leptospirosis. World Health Organization, Geneva, $\mathrm{p} 171$.

Faine, S., Adler, B., Bolin, C. \& Perolat, P. (1999) Leptospira and Leptospirosis, $2^{\text {nd }} \quad$ Edition, Melbourne, Australia: MediSci.

Flegr, J., Havlícek, J., Kodym, P., Malý, M. \& Smahel, Z. (2002) Increased risk of traffic accidents in subjects with latent toxoplasmosis: a retrospective case-control study. BMC Infectious Diseases 2, 11.

Flegr, J., Lenochová, P., Hodný, Z. \& Vondrová, M. (2011) Fatal attraction phenomenon in humans-cat odour attractiveness increased for Toxoplasma-infected men while decreased for infected women. PLoS Neglected Tropical Diseases 5, e1389.

Frenkel, J.K. \& Ruiz, A. (1981) Endemicity of toxoplasmosis in Costa Rica transmission between cats, soil, intermediate hosts and humans. American Journal of Epidemiology 113, 254-269.

Goris, M.G. \& Hartskeerl, R.A. (2013) Leptospirosis serodiagnosis by the microscopic agglutination test. Current Protocols in Microbiology $12 \mathrm{E}-5$.

Goris, M.G.A.; Leeflang, M.M.G, Boer, K.R., Goeijenbier, M., van Gorp, E.C.M., Wagenaar, J.F.P. \& Hartskeerl, R.A. (2012)) Establishment of Valid Laboratory Case Definition for Human Leptospirosis. Journal of Bacteriology and Parasitology 3, 2.

Hill, D. \& Dubey, J.P. (2002) Toxoplasma gondii: transmission, diagnosis and prevention. Clinical Microbiology and Infection 8, 634-640.

Homan, W.L., Vercammen, M., De Braekeleer, J. \& Verschueren, H. (2000) Identification of a 200to 300 -fold repetitive 529 bp DNA fragment in Toxoplasma gondii, and its use for diagnostic and quantitative PCR. International Journal for Parasitology 30, 69-75.

House, P.K., Vyas, A. \& Sapolsky, R. (2011) Predator cat odors activate sexual arousal pathways in brains of Toxoplasma gondii infected rats. PLoS One 6(8), e23277. 
Ingram, W.M., Goodrich, L.M., Robey. E.A. \& Eisen, M.B. (2013) Mice infected with low-virulence strains of Toxoplasma gondii lose their innate aversion to cat urine, even after extensive parasite clearance. PLOS ONE 8(9), e75246.

Jones, J.L., Kruszon-Moran, D., Wilson, M., McQuillan, G., Navin, T. \& McAuley, J.B. (2001) Toxoplasma gondii infection in the United States: seroprevalence and risk factors. American Journal of Epidemiology 154, 357-365.

Khiem, N.T. \& Van Chien, H. (2003) Market study of meat from field rats in the Mekong Delta. Rats, Mice and People: Rodent Biology and Management, 543-547.

Kingdon, J. (1997) The Kingdom Field Guide To African Mammals. London: Academic Press; pp 464.

Kranendork, O., Wolff, J.W., Bohlander, J.W., Roberts, J.M.D., De Geus, A. \& Njenga, R. (1968) Isolation of new Leptospira serovars in Kenya. World Health Organization (WHO) Zoonotics $111,1-5$.

Machang'u, R.S., Mgode, G. \& Mpanduji, D. (1997) Leptospirosis in animals and humans in selected areas of Tanzania. Belgian Journal of Zoology 127, 97-104.

Machang'u, R.S., Mgode, G.F., Assenga, J., Mhamphi, G., Weetjens, B., Cox, C., Verhagen, R., Sondij, S., Goris, M.G. \& Hartskeerl, R.A. (2004) Serological and molecular characterization of Leptospira serovar Kenya from captive African giant pouched rats (Cricetomys gambianus) from Morogoro Tanzania. FEMS Immunology and Medical Microbiology 41, 117121.

Mgode, G.F., Machang'u, R.S., Goris, M.G., Engelbert, M., Sondij, S. \& Hartskeerl, R.A. (2006) New Leptospira serovar Sokoine of serogroup Icterohaemorrhagiae from cattle in Tanzania. International Journal of Systematics and Evolutionary Microbiology 56, 593-597.

Mgode, G.F., Mhamphi, G., Katakweba, A., Paemelaere, E., Willekens, N., Leirs, H., Machang'u, R.S. \& Hartskeerl, R.A. (2005) PCR detection of Leptospira DNA in rodents and shrews from Tanzania. Belgian Journal of Zoology 135, 17-19.

Mohamed, K., Rayah, I.E., Bilal, A., Gumaa, E.T., Magid, A.A., Maly, M. \& Kodym, P. (2009) Immune-diagnosis of latent toxoplasmosis in childbearing age women in rural areas in EL Geizera State, Sudan. International Journal of Medicine and Medical Sciences 1(7), 272-277.

Mwambe, B., Mshana, S.E., Kidenya, B.R., Massinde, A.N., Mazigo, Denna, H.D., Michael, D., Majinge, C. \& Groß, U. (2013) Sero-prevalence and factors associated with Toxoplasma gondii infection among pregnant women attending antenatal care in Mwanza, Tanzania. Parasites \& Vectors 6: 222.

Swai, E.S. \& Schoonman, L. (2009) Seroprevalence of Toxoplasma gondii infection amongst residents of Tanga district in northeast Tanzania. Tanzania Journal of Health Research 11, 205-209.

Swai, E.S. \& Schoonman, L. (2012) A survey of zoonotic diseases in trade cattle slaughtered at Tanga city abattoir: a cause of public health concern. Asian Pacific Journal of Tropical Biomedicine 2, 55-60.

Ter Meulen, J., Lukashevich, I., Sidibe, K., Inapogui , A., Marx , M., Dorlemann, A. Yansane , M. L., Koulemou, K., Chang-Claude, J. \& Schmitz, H. (1996) Hunting of peridomestic rodents and consumption of their meat as possible risk factors for rodent-to-human transmission of Lassa virus in the Republic of Guinea. American Journal of Tropical Medicine and Hygiene $55,661-666$. 